\title{
Mode locking of semiconductor lasers in a coupled-cavity configuration with a strongly coupled empty auxiliary cavity
}

\author{
Ching-Fuh Lin \\ Graduate Institute of Electro-Optical Engineering and Department of Electrical Engineering, \\ National Taiwan University, Taipei, Taiwan
}

Received August 8, 1995; revised manuscript received June 18, 1996

\begin{abstract}
Mode locking of semiconductor lasers in a coupled-cavity configuration with an empty auxiliary cavity is studied theoretically. The empty auxiliary cavity, when strongly coupled to the main cavity, is found to influence the pulsation situation significantly. The predicted pulse widths and chirp parameters in the steady state are in good agreement with the experimental measurements. The influences of the linear phase shift induced by the cavity-length variation and the self-phase modulation in the main cavity on the pulsation are also briefly discussed. (C) 1997 Optical Society of America. [S0740-3224(97)02601-5]
\end{abstract}

A coupled-cavity configuration is an effective way of generating ultrashort optical pulses. ${ }^{1,2}$ The schemes involving coupled-cavity configurations have been termed coupled-cavity, additive-pulse, and interferential mode locking. ${ }^{3}$ The coupled-cavity configuration is used to augment the original laser cavity with an auxiliary cavity that contains a nonlinear optical element. An optical fiber is usually used as the nonlinear optical element to force the optical pulses to undergo significant self-phase modulation (SPM), which induces different phase shifts at the wings and the center of the pulses. As the length of the auxiliary cavity is adjusted to match that of the main cavity so that destructive interference occurs at the wings of the overlapped pulse, the pulse is shortened. The SPM in the auxiliary cavity is essential for the pulseshortening effect. However, it should be reasonable to assert that the required SPM can be placed in the main cavity and also that an empty auxiliary cavity will assist pulse shortening. Although an additional nonlinear element is seldom used in the main cavity, because it will then raise the lasing threshold, the existing element in the main cavity might exhibit SPM. Therefore this paper will explore the pulse-shortening effect caused by an empty auxiliary cavity because the main cavity already has SPM.

Without the insertion of any additional nonlinear elements in the main cavity, an empty auxiliary cavity has been reported to generate short optical pulses. ${ }^{4-6}$ The short pulses were generated only with the auxiliary mirror in motion, which in explanation was said to introduce a continuously changing phase shift in the feedback path. ${ }^{7}$ The induced frequency shifting then prevents the dominance of a single frequency and the operation of continuous oscillation, resulting in a response that resembles mode locking. The empty auxiliary cavity ${ }^{4-6}$ offers one simple and effective way of generating ultrashort pulses. However, the mode locking is not sustained steadily, and so the auxiliary mirror needs to be driven to move peri- odically. The mode locking builds up and decays accordingly. On the other hand, with the auxiliary mirror fixed at a given position, an empty auxiliary cavity has also been reported to significantly shorten the pulses generated from an external-cavity semiconductor laser. ${ }^{8}$ In this experiment a significant amount of SPM is suspected to exist in the monolithically integrated absorber in the laser-diode chip and to play an important role in the pulse-shortening effect. With the SPM in the main cavity, this configuration behaves, as a matter of fact, more like the traditional coupled cavities with a nonlinear auxiliary cavity than like those with a moving auxiliary mirror. A quantitative study of mode locking in such a coupled-cavity configuration with an empty auxiliary cavity fixed at a given position is presented in this paper. With the existing SPM in the main cavity, this coupledcavity mode-locking technique provides an even simpler way of generating ultrashort pulses than previous techniques ${ }^{4-6}$ with a moving mirror.

A model starting from those that were previously used to explain additive-pulse mode locking with a nonlinear auxiliary cavity $^{9-11}$ is developed. Previous models ${ }^{10,11}$ of additive-pulse mode locking approximate the coupling of the two pulses to yield a simple formulation for the coupled-cavity configuration because only weak feedback is returned to the main cavity. In this paper no approximation is made for the coupling. A simple formulation is still obtained for the coupled-cavity configuration even when the main cavity has strong feedback from the auxiliary cavity, which could happen if the cleaved facet of a laser diode is used as the coupling mirror. ${ }^{8,12}$ A master equation similar to the one in Ref. 11 is derived. Practical values of the parameters in the master equation are chosen to predict the pulsation of the semiconductor lasers in the coupled-cavity configuration with an empty auxiliary cavity. Theoretical calculations are compared with the experimental measurements for different amounts of feedback from the empty auxiliary cavity. 
The influences of the cavity-length induced phase shift and the SPM in the main cavity on the pulsation are discussed briefly.

Figure 1 shows a schematic of the coupled-cavity configuration for analysis. The main cavity on side $a$, containing the gain material such as a semiconductor laser, is formed between mirror $\mathrm{M}_{1}$ and the coupling mirror. The auxiliary cavity on side $b$ is formed between mirror $\mathrm{M}_{2}$ and the coupling mirror. The auxiliary cavity is empty. It is assumed that mirror $\mathrm{M}_{1}$ has a reflectivity of $100 \%$, the coupling mirror has a field reflectivity $r$, and mirror $\mathrm{M}_{2}$ returns the optical field with an attenuation factor $L$. The coupling mirror can be the cleaved facet of the laser diode. Consider the incident and the reflected wave amplitudes $A_{k}, B_{k}$, and $A_{k}^{\prime}, B_{k}^{\prime}$ at the coupling mirror separating the main cavity and the auxiliary cavity. These four wave amplitudes at the coupling mirror are related by the following two equations:

$$
\begin{aligned}
& A_{k}^{\prime}=r A_{k}+\sqrt{1-r^{2}} B_{k}, \\
& B_{k}^{\prime}=\sqrt{1-r^{2}} A_{k}-r B_{k} .
\end{aligned}
$$

On side $a$, the time envelope $A_{k}^{\prime}$ of the wave leaving the coupling mirror passes through the semiconductor laser gain medium and returns from mirror $\mathrm{M}_{1}$. This passage changes the wave amplitude $A_{k}^{\prime}$ to $A_{k+1}$ before it meets the coupling mirror again. Therefore

$$
A_{k+1}=\exp \left[-j\left(\phi_{1}+\Phi_{1}\right)\right] A_{k}^{\prime},
$$

where $\exp \left(-j \phi_{1}\right)$ is the linear phase-shift term and where $\exp \left(-j \Phi_{1}\right)$ includes gain, loss, group-velocity dispersion, saturable absorption action, and SPM. For simplicity of discussion, they are assumed to be small enough that $\exp \left(-j \Phi_{1}\right) \sim 1-j \Phi_{1}$. Then $A_{k+1}$ can be represented by the following equation:

$$
\begin{aligned}
A_{k+1}= & \exp \left(-j \phi_{1}\right)\left[1-l_{m}+g_{m}\left(1+\frac{1}{\omega_{g}^{2}} \frac{\mathrm{d}^{2}}{\mathrm{~d} t^{2}}\right)\right. \\
& \left.+j D_{m} \frac{\mathrm{d}^{2}}{\mathrm{~d} t^{2}}+\left(\gamma_{m}-j \delta_{m}\right)\left|A_{k}^{\prime}\right|^{2}\right] A_{k}^{\prime},
\end{aligned}
$$

where $g_{m}$ and $D_{m}$ are the gain and the dispersion of the semiconductor laser-diode chip, respectively; $\omega_{g}$ is the gain bandwidth and $l_{m}$ is the loss in the main cavity; $\gamma_{m}\left|A_{k}^{\prime}\right|^{2}$ represents the saturable absorption action of the integrated absorber; and $-j \delta_{m}\left|A_{k}^{\prime}\right|^{2}$ represents the influence of SPM.

On side $b$, the wave amplitude $B_{k}^{\prime}$ is returned by mirror $M_{2}$. The returned wave amplitude $B_{k+1}$ does not change its shape except that it is attenuated by a factor $L$ and

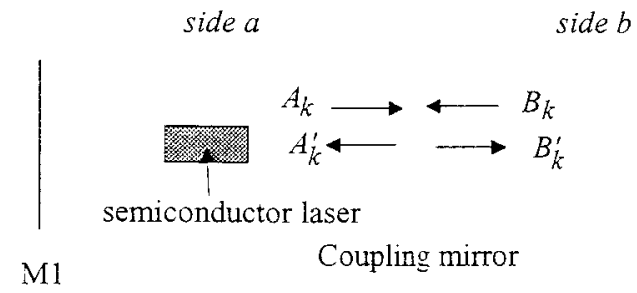

Fig. 1. Schematic of the coupled-cavity configuration. also experiences a linear phase shift $\phi_{2} . \phi_{2}=4 \pi l_{2} / \lambda$, where $l_{2}$ is the length of the auxiliary cavity. Therefore $B_{k+1}$ is related to $B_{k}^{\prime}$ by

$$
B_{k+1}=L \exp \left(-j \phi_{2}\right) B_{k}^{\prime} \text {. }
$$

In the steady state the pulse shape is not changed by each round trip, so $A_{k+1}=A_{k}$ and $B_{k+1}=B_{k}$. Then $A_{k}^{\prime}$ and $A_{k}$ can be simply related by

$$
A_{k}^{\prime}=\alpha \exp (j \theta) A_{k},
$$

where

$$
\alpha \exp (j \theta)=\frac{L+r \exp \left(j \phi_{2}\right)}{r L+\exp \left(j \phi_{2}\right)} .
$$

One can then substitute $A_{k}^{\prime}$ from Eq. (5) into Eq. (3) and obtain a general equation for the coupled-cavity configuration with an empty auxiliary cavity:

$$
\begin{aligned}
A_{k}= & \alpha\left[1-l_{m}+g_{m}\left(1+\frac{1}{\omega_{g}^{2}} \frac{\mathrm{d}^{2}}{\mathrm{~d} t^{2}}\right)+j D_{m} \frac{\mathrm{d}^{2}}{\mathrm{~d} t^{2}}\right. \\
& \left.+\alpha^{2}\left(\gamma_{m}-j \delta_{m}\right)\left|A_{k}\right|^{2}\right] \exp \left[j\left(\theta-\phi_{1}\right)\right] A_{k} .
\end{aligned}
$$

Special care is required to handle the linear phase-shift term $\exp \left[j\left(\theta-\phi_{1}\right)\right]$. In cw operation the derivatives and the intensity-dependent terms have no effects and so are set to zero. To have a nonzero solution of $A_{k}$ in cw oscillation, the net phase shift needs to be set to zero (or multiples of $2 \pi$ ), $\theta-\phi_{1}=2 m \pi$, with $m$ an integer. This condition determines the cw oscillation frequency of the coupled-cavity configuration. The pulse operation is treated approximately as an expansion of the system from $\mathrm{cw}$ operation. The net phase shift is kept at $2 m \pi$, but the derivatives and the intensity-dependent terms are retained. The influence of the net phase shift different from $2 m \pi$ will be discussed below. With the net phase shift set to $2 m \pi$, Eq. (7) becomes

$$
\begin{aligned}
{\left[1-\frac{1}{\alpha}-l_{m}+g_{m}(\right.} & \left.+\frac{1}{\omega_{g}{ }^{2}} \frac{\mathrm{d}^{2}}{\mathrm{~d} t^{2}}\right)+j D_{m} \frac{\mathrm{d}^{2}}{\mathrm{~d} t^{2}} \\
& \left.+\alpha^{2}\left(\gamma_{m}-j \delta_{m}\right)\left|A_{k}\right|^{2}\right] A_{k}=0 .
\end{aligned}
$$

In the above equation, $\alpha$ is involved with $l_{2}$, the length of the empty auxiliary cavity, which therefore modifies the laser parameters such as the loss $\left(1 / \alpha-1+l_{m}\right)$, the saturable-absorption action $\left(\alpha^{2} \gamma_{m}\right)$, and the $\operatorname{SPM}\left(\alpha^{2} \delta_{m}\right)$. In addition, because the value of $\alpha$ varies periodically with the phase shift $\phi_{2}$ [from Eq. (6)], the periodic variation of the pulsation situation with the length of the auxiliary cavity can be expected. It should be noted that, if the coupling is weak ( $L \ll 1$ ), the phase shift $\phi_{2}$ has only a slight influence on the value of $\alpha$, and so the pulse shape is negligibly changed. However, the empty auxiliary cavity should still provide a stabilizing influence. ${ }^{11,13}$

An ansatz solution has already been introduced for the master equation $(8)^{11}$ :

$$
A_{k}=A[\operatorname{sech}(t / \tau)]^{(1+j \beta)},
$$

where the pulse width $\tau$ and the chirp parameter $\beta$ are given by

$$
\tau=\left(2 g_{m} / W \omega_{g}^{2}\right) \tau_{n},
$$




$$
\beta=-\chi \pm \sqrt{\chi^{2}+2}
$$

with

$$
\begin{gathered}
\tau_{n}=-\frac{3 \beta\left(1+D_{n}^{2}\right)}{\alpha^{2}\left(\gamma_{m} D_{n}+\delta_{m}\right)}, \quad \chi=\frac{3}{2} \frac{\delta_{m} D_{n}-\gamma_{m}}{2 \gamma_{m} D_{n}+\delta_{m}}, \\
D_{n}=\frac{\omega_{g}{ }^{2}}{g_{m}} D_{m} .
\end{gathered}
$$

The plus in Eq. (11) is used if $\left(\gamma_{m} D_{n}+\delta_{m}\right)<0$, and the minus is used if $\left(\gamma_{m} D_{n}+\delta_{m}\right)>0$. This theory does not account for the full gain-saturation dynamics during the pulse. Instead a steady-state gain saturation is used, and so, the gain $g_{m}$ may be determined by requiring it to balance the loss $\left(g_{m}=1 / \alpha-1+l_{m}\right){ }^{11}$ It is also assumed that the gain saturation can be described by the formula ${ }^{11}$

$$
g_{m}=\frac{g_{o}}{1+W / W_{s}},
$$

where $W=2 A^{2} \tau$ is the energy of the pulse and $W_{s}$ is the effective saturation energy. The pulse formula given by Eq. (9) states that the pulse is chirped with a chirp parameter $\beta$. With the chirp eliminated, the pulse width can be further shortened to

$$
\tau_{\mathrm{cm}}=\tau / \sqrt{1+\beta^{2}} .
$$

$\tau_{\mathrm{cm}}$ denotes the compressed pulse width. The influences of group-velocity dispersion, saturable absorption action, and SPM on the pulse shape are similar to those of the analysis in Ref. 11 because the same master equation is followed. This particularly addresses the significance of the empty auxiliary cavity. Equation (8) shows that the empty auxiliary cavity influences the pulsation situation through three parts: the loss factor $\left(1 / \alpha-1+l_{m}\right)$, the saturable absorption action $\left(\alpha^{2} \gamma_{m}\right)$, and the SPM $\left(\alpha^{2} \delta_{m}\right)$. The values of these parameters in a reasonable range will be chosen to predict the pulsation of the semiconductor lasers in the coupled-cavity configuration with an empty auxiliary cavity.

The dispersion parameter $D_{m}$ is directly proportional to $\mathrm{d}^{2} n / \mathrm{d} \lambda^{2}$, which is in the range of $20-50 \mu \mathrm{m}^{-2}$ for AlGaAs-GaAs semiconductors. ${ }^{14} \mathrm{~A} \mathrm{~d}^{2} n / \mathrm{d} \lambda^{2}$ of $45 \mu \mathrm{m}^{-2}$ is used for the calculation. The loss $\left(l_{m}\right)$ is assumed to be $10 \%$ to account for the coupling loss from the laser diode to the main cavity. The SPM parameter $\delta_{m}$ equals $\left(\omega_{o} / c\right)\left(n_{2} d_{a} / A_{\text {eff }}\right)$, where $n_{2}$ is the nonlinear index, $A_{\text {eff }}$ is the effective cross section of the mode in the waveguide of the laser diode, and $d_{a}$ is the length of the monolithically integrated absorber, which is $30 \mu \mathrm{m}$ long in the experiment. $^{8}$ Because the gain region is externally pumped, the carrier density is kept approximately constant. Therefore optically induced changes in the refractive index in response to variations in the carrier density occur mainly in the unpumped absorber, and so the SPM is only considered in the integrated absorber. The value of $n_{2}$ for semiconductors is estimated to be $10^{-9} \mathrm{~cm}^{2} / \mathrm{W}$ when the pulse width is much larger than the carrier life time, ${ }^{15}$ which is $\sim 400 \mathrm{ps}$. In the case here, because the pulse is much shorter, the carriers can hardly respond to the pulse, and so $n_{2}$ should be much less than $10^{-9} \mathrm{~cm}^{2} / \mathrm{W}$ but still larger than $10^{-14} \mathrm{~cm}^{2} / \mathrm{W}$ for the optical Kerr effect. A medium value that leads to $\delta_{m} \sim 0.1 \mathrm{~W}^{-1}$ is used.

The description of the saturable absorber as an instantaneous function of the intensity in Eq. (7) limits this theory to the cases of fast saturable absorbers. It will be shown that use of the instantaneous response of the absorption action could well predict the mode-locked pulses in Ref. 8, indicating that the devices therein might contain a fast response of the absorption recovery. Yang and Gopinath $^{16}$ had also concluded from their simulation that a short absorber recovery time is important for the stable operation of passively mode-locked semiconductor lasers. Because the devices in Ref. 8 could be electrically pumped only in pulsed condition, they could possibly suffer the aging effect, and defects might be induced in the operation, leading to fast absorption recovery. Therefore fast absorption recovery is assumed in the model to make the analytical solution possible even with the strongly coupled auxiliary cavity. Even so, the estimation of the value of the saturable absorption action $\gamma_{m}$ remains difficult. In the simulation the calculated pulse width generated from the external-cavity laser without the auxiliary cavity dramatically changes with the value of $\gamma_{m}$. For $\gamma_{m}=0$, the pulse width is larger than the round-trip time, indicating no pulsation. Increasing $\gamma_{m}$ will eventually result in subpicosecond pulses from the calculation. In the experiment of semiconductor lasers with a monolithically integrated absorber in an external cavity, but without the auxiliary cavity, the pulse width is approximately between 20 and 30 ps. $^{8}$ To match this experimental result, $\gamma_{m}$ is approximated as $0.03 \mathrm{~W}^{-1}$, which results in a pulse width of $26 \mathrm{ps}$ under a reasonable assumption of $W \sim 2 \mathrm{pJ}^{17}$ and a gain bandwidth of $30 \mathrm{~nm}$.

With these values applied to the master equation for the coupled-cavity configuration with an empty auxiliary cavity, the pulse width in the steady state is calculated. By varying of the phase shift $\phi_{2}$, the calculated pulse width changes periodically as expected. The minimum pulse width is obtained when $\alpha$ is maximum, occurring as $\phi_{2}$ is a multiple of $2 \pi$. The calculated pulse widths at $\phi_{2}=0$ and the corresponding chirp parameters are given in Table 1 for different $M_{2}$ reflectivities. In the experiment the optimal distance between the grating and the retroreflector for pulse compression is not obviously dependent on the $M_{2}$ reflectivity, indicating that the chirp of the uncompressed pulses for different $M_{2}$ reflectivities is similar. Table 1 does show that the chirp parameter is almost the same for different $\mathrm{M}_{2}$ reflectivities.

Table 1. Calculated Compressed Pulse Widths at $\phi_{2}=0$ and Corresponding Chirp Parameters for Different $M_{2}$ Reflectivities

\begin{tabular}{ccc}
\hline $\begin{array}{c}\mathrm{M}_{2} \\
\text { Reflectivity } \\
(L \times L)\end{array}$ & $\begin{array}{c}\text { Chirp } \\
\text { Parameter } \\
\beta\end{array}$ & $\begin{array}{c}\text { Compressed } \\
\text { FWHM } \\
\left(2 \tau_{\mathrm{cm}} \times 0.88\right)(\mathrm{ps})\end{array}$ \\
\hline 0.2 & -8.77 & 0.69 \\
0.3 & -8.73 & 0.52 \\
0.4 & -8.67 & 0.41 \\
0.5 & -8.59 & 0.33 \\
\hline
\end{tabular}


The above analysis shows that in the steady state the empty auxiliary cavity effectively changes the reflectivity $\alpha$, which equals $r$ without the auxiliary cavity. In addition, the minimum pulse width occurs as $\phi_{2}$ is a multiple of $2 \pi$. The oscillation frequencies for the pulse operation then might be determined by the strongly coupled empty auxiliary cavity. If the linear phase shift $\phi_{1}$ in the main cavity deviates from $2 m \pi$, the laser parameters in Eq. (8) could be further changed by the multiplication of this linear phase-shift term with those parameters in the first bracket of Eq. (7). Obviously the resulting laser parameters and the pulsation situation also vary periodically with the phase shift $\phi_{1}$. Simulation shows that for $\phi_{1}$ slightly more than zero the pulse width is further reduced. The shortest pulse is approximately at $\phi_{1}$ $=0.1 \pi$, which is slightly changed by the amount of the feedback from the auxiliary cavity. Very short pulse widths are also obtained for $\phi_{1}$ approximately from 0 to $0.3 \pi$. Beyond this range, the pulse width increases significantly. Figure 2 shows the variation of the compressed pulse width with the phase shift $\phi_{1}$ for different amounts of the feedback. The pulse widths for $\phi_{1}$ from $\sim 0$ to $0.3 \pi$ still spread over a certain range, as listed in Table 2. The experiment ${ }^{8}$ therefore tended to measure pulse widths in this range. The experimental pulse widths from Ref. 8 are also listed in Table 3 for comparison, which shows that the calculations from the model are in good agreements with the experiments. Figure 2 also shows that another range of relatively short pulses is predicted for $\phi_{1}$ sightly more than $\pi$. The second range of short pulses should be expected from Eqs. (1) and (2), which imply that the pulse-shortening effect could occur either in the main cavity or in the auxiliary cavity, as $A_{k}$ and $B_{k}$ have a phase difference $\pi$ or $2 \pi$.

To show that SPM in the main cavity is an important factor for the pulse-shortening effect in the coupled-cavity configuration with an empty auxiliary cavity, different amounts of SPM are applied to simulate the pulsation. If SPM is increased to make $\delta_{m} 10$ times larger $\left(\delta_{m}\right.$

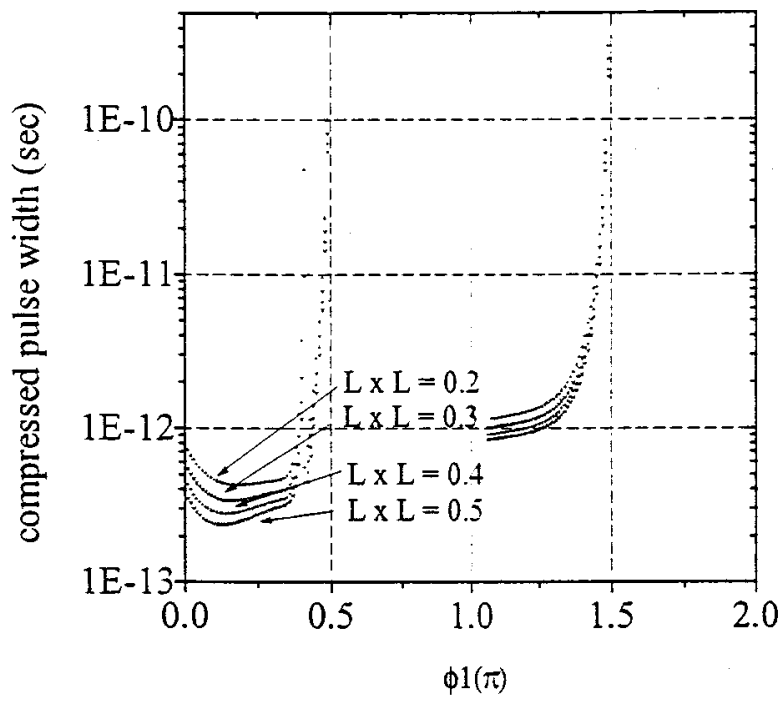

Fig. 2. Compressed pulse width versus phase $\phi_{1}$ for different amounts of feedback from the empty auxiliary cavity.
Table 2. Calculated Compressed Pulse Widths for Different Amounts of Feedback from the Empty Auxiliary Cavity with $\phi_{1}$ Approximately in the Range 0-0.3 $\pi$

\begin{tabular}{cc}
\hline $\begin{array}{c}\mathrm{M}_{2} \\
\text { Reflectivity } \\
(L \times L)\end{array}$ & $\begin{array}{c}\text { Compressed } \\
\text { FWHM } \\
\left(2 \tau_{\mathrm{cm}} \times 0.88\right)(\mathrm{ps})\end{array}$ \\
\hline 0.2 & $0.38-0.69$ \\
0.3 & $0.30-0.52$ \\
0.4 & $0.25-0.41$ \\
0.5 & $0.21-0.33$ \\
\hline
\end{tabular}

Table 3. Measured Compressed Pulse Widths for Different $M_{2}$ Reflectivities

\begin{tabular}{ccc}
\hline Device & $\begin{array}{c}\mathrm{M}_{2} \text { Effective Reflectivity } \\
\left(R_{2} \times 0.64\right)\end{array}$ & $\begin{array}{c}\text { Compressed } \\
\text { FWHM } \\
(\mathrm{ps})\end{array}$ \\
\hline$E-1-12$ & 0.19 & $0.39-0.70$ \\
& 0.32 & $0.38-0.66$ \\
$E-3-12$ & 0.37 & $0.30-0.49$ \\
& 0.37 & $0.30-0.51$ \\
& 0.48 & $0.19-0.42$ \\
\hline
\end{tabular}

Table 4. Pulse Widths ${ }^{a}$

\begin{tabular}{cccc}
\hline$\delta_{m}\left(\mathrm{~W}^{-1}\right)$ & $\tau_{1}(\mathrm{ps})$ & $\tau_{2}(\mathrm{ps})$ & $\tau_{\mathrm{cm}}(\mathrm{ps})$ \\
\hline 0.1 & 26 & 1.25 & 0.21 \\
1 & 32 & 0.45 & 0.03 \\
\hline
\end{tabular}

${ }^{a} \tau_{1}$, pulse width without the empty auxiliary cavity; $\tau_{2}$, pulse width with the empty auxiliary cavity before pulse compression; $\tau_{\mathrm{cm}}$, pulse width with the empty auxiliary cavity after pulse compression.

$=1 \mathrm{~W}^{-1}$ ), with the other parameters remaining at the same values, the pulse width without the empty auxiliary cavity is approximately the same (32 ps). However, the empty auxiliary cavity could shorten the pulse width to as short as $0.45 \mathrm{ps}$, and the pulse width could be further reduced to $30 \mathrm{fs}$ as the chirp is eliminated. A comparison of the pulse widths for $\delta_{m}=01 \mathrm{~W}^{-1}$ and $\delta_{m}=1 \mathrm{~W}^{-1}$ given in Table 4 clearly shows the significance of SPM.

In conclusion, mode-locking of semiconductor lasers in a coupled-cavity configuration with an empty auxiliary cavity is studied theoretically. With the empty auxiliary cavity a simple formulation can be obtained for the coupled-cavity configuration even with a strong coupling between the auxiliary cavity and the main cavity. Calculations of the minimum pulse widths and the corresponding chirp parameters in the steady state, based on this model, are in good agreement with the experimental measurements of mode-locked semiconductor lasers in a coupled-cavity configuration. With the strongly coupled empty auxiliary cavity the shortest pulse width is obtained when the phase shift induced by the auxiliary cavity is a multiple of $2 \pi$ and the linear phase shift induced by the main cavity is slightly more than $2 m \pi$. The pulses are also expected to be further shortened with increasing SPM in the main cavity. 


\section{ACKNOWLEDGMENTS}

This work was performed in part at the School of Electrical Engineering at Cornell University. C. L. Tang's suggestions are greatly acknowledged. The author also thanks colleagues in Tang's group and Bor-Lin Lee at the Graduate Institute of Electro-Optical Engineering at the National Taiwan University for their helpful discussions. This work is supported by the National Science Council, Taipei, Taiwan, under contract NSC83-0417-E-002-011.

\section{REFERENCES}

1. J. Mark, L. Y. Liu, K. L. Hall, H. A. Haus, and E. P. Ippen, "Femtosecond pulse generation in a laser with a nonlinear external resonator," Opt. Lett. 14, 48 (1989).

2. C. P. Yakymyshyn, J. F. Pinto, and C. R. Pollock, "Additivepulse mode-locked $\mathrm{NaCl}: \mathrm{OH}^{-}$laser," Opt. Lett. 14, 621 (1989).

3. W. Sibbett, "Hybrid and passive mode locking in coupledcavity lasers," Ultrafast Phenomena 7, 2 (1990).

4. P. M. W. French, S. M. J. Kelly, and J. R. Taylor, "Mode locking of a continuous-wave titanium-doped sapphire laser using a linear external cavity," Opt. Lett. 15, 378 (1990).

5. P. G. Wigley, P. M. W. French, and J. R. Taylor, "Mode locking of a continuous wave neodymium doped fiber laser with a linear external cavity," Electron. Lett. 26, 1283 (1990).

6. Y. Shi, C. V. Poulsen, M. Sejka, and O. Poulsen, "Modelocked $\mathrm{Pr}^{3+}$-doped silica fiber laser with an external cavity," J. Lightwave Technol. 12, 749 (1994).
7. C. C. Cutler, "Why does linear phase shift cause mode locking?" IEEE J. Quantum Electron. 28, 282 (1992).

8. C. F. Lin and C. L. Tang, "Generation of ultrashort pulses from a superluminescent diode with a monolithically integrated absorber in a coupled-cavity configuration," Appl. Phys. Lett. 63, 2594 (1993).

9. K. J. Blow and David Wood, "Mode-locked lasers with nonlinear external cavities," J. Opt. Soc. Am. B 5, 629 (1988).

10. E. P. Ippen, H. A. Haus, and L. Y. Liu, "Additive pulse mode locking," J. Opt. Soc. Am. B 6, 1736 (1989).

11. H. A. Haus, J. G. Fujimoto, and E. P. Ippen, "Structures for additive pulse mode locking," J. Opt. Soc. Am. B 8, 2068 (1991).

12. W. H. Loh and C. F. Lin, "Optical pulse generation with a semiconductor laser in a coupled-cavity configuration," Pure Appl. Opt. 1, 181 (1992).

13. G. H. C. New, "Self-stabilization of synchronously modelocked lasers," Opt. Lett. 15, 1306 (1990).

14. Y. Silberberg and P. W. Smith, "Subpicosecond pulses from a mode-locked semiconductor laser," IEEE J. Quantum Electron. 22, 759 (1986).

15. G. P. Agrawal and N. A. Olsson, "Self-phase modulation and spectral broadening of optical pulses in semiconductor laser amplifiers," IEEE J. Quantum Electron. 25, 2297 (1989).

16. W. Yang and A. Gopinath, "Study of passive mode locking of semiconductor lasers using time-domain modelling," Appl. Phys. Lett. 63, 2717 (1993).

17. D. J. Derickson, R. J. Helkey, A. Mar, J. R. Karin, J. E. Bowers, and R. L. Thornton, "Suppression of multiple pulse formation in external-cavity mode-locked semiconductor lasers using intrawaveguide saturable absorbers," IEEE Photonics Technol. Lett. 4, 333 (1992). 